\title{
"La Educación Superior y su compromiso con el Desarrollo Sostenible" Caso: Contribución de la Universidad Nacional de Pilar al Desarrollo Sostenible en el Departamento de Ñeembucú"
}

\author{
MSc. Luisa Del Pilar Gamarra Z. \\ lucy-g@hotmail.es \\ Universidad Nacional de Pilar
}

\section{RESUMEN}

Según el Informe Brundtlan (1987), Desarrollo Sostenible es aquel que garantiza las necesidades del presente sin comprometer las posibilidades de las generaciones futuras para satisfacer sus propias necesidades. Ibañez (1994), menciona que la Educación Superior es la Institución tiene como objetivo la formación de capacidades y actitudes de los individuos para su integración a la sociedad como seres capaces de regular el status quo y a la vez puedan transformar la realidad social en pos de los valores vigentes en un momento histórico determinado. La tarea de la educación superior es "la formación de profesionales competentes; individuos que resuelvan creativamente, es decir, de manera novedosa, eficiente y eficaz, los problemas sociales. El Trabajo de Investigación se plantea como objetivo general "Determinar de qué manera la Universidad Nacional de Pilar, como Institución de Educación Superior, contribuye al desarrollo sostenible en el Departamento de Ñeembucú, desde sus funciones sustantivas, atendiendo su visión, misión y política institucional", responde a un estudio de caso, el tipo de estudio es descriptivo, de corte transversal, implementa el método cualitativo y cuantitativo. Los resultados permitirán identificar y describir el aporte de la UNP al desarrollo sostenible atendiendo las funciones sustantivas de la IES y los objetivos planteados en el Plan 2030.

Palabras claves: Educación Superior- Desarrollo Sostenible - Plan 2030- Funciones Sustantiva 


\title{
"Higher Education and its commitment to Sustainable Development" Case: Contribution of the National University of Pilar to Sustainable Development in the Department of Neembucú"
}

\begin{abstract}
Brundtlan Report (1987), Sustainable Development is one that guarantees the needs of the present without compromising the possibilities of future generations to satisfy their own needs. Ibañez (1994), mentions that Higher Education is the Institution has as its objective the formation of capacities and attitudes of individuals for their integration into society as beings capable of regulating the status quo and at the same time they can transform social reality in pursuit of the values in force at a given historical moment. The task of higher education is "the training of competent professionals; individuals who creatively solve, that is, in a novel, efficient and effective way, social problems. The general objective of the Research Work is "To determine how the National University of Pilar, as an Institution of Higher Education, contributes to sustainable development in the Department of Ñeembucú, from its substantive functions, attending to its vision, mission and institutional policy", responds to a case study, the type of study is descriptive, crosssectional, implements the qualitative and quantitative method. The results will make it possible to identify and describe the contribution of the UNP to sustainable development, taking into account the substantive functions of the IES and the objectives set out in the 2030 Plan.
\end{abstract}

Keywords: Higher Education- Sustainable Development - Plan 2030- Substantive Functions.

Artículo recibido: 05 de Abril 2021 Aceptado para publicación: 28 de Mayo 2021

Correspondencia: lucy-g@hotmail.es Conflictos de Interés: Ninguna que declarar 


\section{INTRODUCCIÓN}

El informe sobre Desarrollo Mundial del Grupo Banco Mundial (2018), dice que la educación contribuye significativamente al logro de los objetivos estratégicos, poner fin a la pobreza extrema, y promover la prosperidad compartida, además elevan las aspiraciones, generan valores y principalmente, enriquecen la vida de las personas. (Banco Desarrollo Mundial. 2018).

La Agenda 2030 de Desarrollo Sostenible presenta a la educación como la base para mejorar la vida y el desarrollo sostenible. Además de mejorar la calidad de vida de las personas, en el acceso a la educación inclusiva y equitativa, puede ayudar a abastecer a la población local con las herramientas necesarias para desarrollar soluciones innovadoras a los problemas más grandes del mundo. (Agenda 2030. 2015)

En base a los objetivos del desarrollo sostenible, en el plan 2030 y en las normativas que rigen a la Universidad Nacional de Pilar, que desde sus orígenes en coincidencia con el despertar democrático en nuestro país, por voluntad de todos sus estamentos manifestada en asamblea universitaria, convencidos de que la educación es un compromiso social y elemento esencial de la democracia, el desarrollo sostenible y la paz, e interpretando la historia de la UNP y su identidad definida por la voluntad popular, establecen sublimes objetivos como la de garantizar la participación ciudadana, garantizar la calidad en la formación profesional, la investigación científica y la extensión universitaria, propender al crecimiento armónico de la universidad, custodiando su área de influencia, preservando el ambiente y el patrimonio histórico y cultural del departamento y de la Nación, entre otros. (Estatuto UNP. 2015).

Atendiendo estos antecedentes la Universidad Nacional de Pilar se propone como misión, formar profesionales críticos, creativos, con principios éticos, conocimientos científicos, capacidad tecnológica y artística como ciudadanos protagonistas en una institución inclusiva, solidaria e internacionalizada, comprometida con el desarrollo sostenible mediante la docencia, la investigación, la tecnología y la extensión. (Plan estratégico UNP. 2015).

\section{Desarrollo Sostenible}

Según el informe Brundtland (Naciones Unidas, 1987, pág. 23), Desarrollo Sostenible es aquel que garantiza las necesidades del presente sin comprometer las posibilidades de las generaciones futuras para satisfacer sus propias necesidades. La agenda 2030 de 
desarrollo sostenible (2015), menciona que la educación es la base para mejorar nuestra vida y el desarrollo sostenible. Además de mejorar la calidad de vida de las personas, el acceso a la educación inclusiva y equitativa puede ayudar abastecer a la población local con las herramientas necesarias para desarrollar soluciones innovadoras a los problemas más grandes del mundo. (Agenda 2030. 2015).

El Departamento de Ñeembucú por mucho tiempo se mantuvo aislada del centro económico y político del país, principalmente por la falta de camino de todo tiempo y la falta de una Institución de Educación Superior que permita a los jóvenes continuar sus estudios universitarios en su departamento y contribuir al crecimiento y desarrollo sostenible en el departamento. La falta de una IES, ocasionaba que solo una minoría de jóvenes pudieran tener acceso a una educación superior fuera del departamento o del país incluso, separándose de sus familias y con muchas limitaciones en cuanto a comunicación. En el año 1991, luego de finalizar un periodo de gobierno dictatorial, se crea la Universidad de Pilar y forma parte de un periodo de democratización de la educación, instalándose en la ciudad de Pilar, capital del departamento de Ñeembucú que posteriormente por Ley $\mathrm{N}^{\circ} 529$ del año 1994 se convierte en Universidad Nacional de Pilar.

La Universidad Nacional de Pilar cuenta con un Estatuto en el cual y convencidos de que la educación es un compromiso social y uno de los elementos fundamentales de la democracia y desarrollo sostenible y la paz, se plasman objetivos sublimes como la de garantizar la participación ciudadana, garantizar la calidad de la formación profesional, la investigación científica y la extensión universitaria, propender al crecimiento armónico de la universidad, custodiando su área de influencia, preservando el ambiente entre otros (Estatuto UNP.2015). En su Plan Operativo (2015), hace mención a su visión como IES referente por su excelencia académica, reconocida por la producción científica y tecnológica en el ámbito local, nacional e internacional, con una gestión participativa e innovadora, a través de talentos humanos comprometidos y éticos, en interacción con el contexto social y ecológico y su misión, la de formar profesionales críticos, creativos, con principios éticos, conocimientos científicos, capacidad tecnológica y artística como ciudadanos protagonistas en una institución inclusiva, solidaria e internacionalizada, comprometida con el desarrollo sostenible mediante la docencia, la investigación, la tecnología y la extensión.( Plan Operativo UNP. 2015). Estos antecedentes muestran que 
la Universidad Nacional de Pilar desde su génesis, creada por voluntad popular o llamémosle compromiso social, preocupados por la formación de los jóvenes del departamento, apuestan a la Educación Superior como base fundamental del desarrollo sostenible, para mejorar la calidad de vida de su población, mediante las funciones esenciales de la E.S.

La vinculación de la universidad con la sociedad, la responsabilidad y pertinencia social, el involucramiento de la sociedad civil a los fines y objetivos institucionales, son estrategias que permitirán a la universidad contribuir al desarrollo sostenible mediante acciones tendientes a mejorar la calidad de vida de los habitantes del Departamento de Ñeembucú.

\section{Caracterización del Departamento de Ñeembucú.}

Los datos e informaciones presentadas en este apartado son extraídos de Atlas Censal Paraguay, que reúne información geográfica y estadística actualizada de 17 departamentos de la República del Paraguay y sus distritos. Atlas Censal del Paraguay es un material elaborado y publicado por la Dirección General de Estadística, Encuestas y Censos (DGEEC), dependiente de la Secretaría Técnica de Planificación del Desarrollo Económico y Social de la Presidencia de la República. Las informaciones presentadas provienen de los Censos Nacionales de Población y Viviendas y principalmente del último realizado en el año 2002, así como de otras fuentes de organismos del Gobierno. El departamento de Neembucú se ubica en el extremo suroeste de la región Oriental, ocupando un área entre los paralelos $25^{\circ} 35^{\prime}$ y $27^{\circ} 20^{\prime}$ de latitud sur y los meridianos $56^{\circ} 35^{\prime}$ y $58^{\circ} 40^{\prime}$ de longitud oeste, al norte limita con Central y al este con Paraguarí y Misiones; se separa de la República Argentina al sur mediante el río Paraná y al oeste a través del río Paraguay.

El departamento de Ñeembucú según el censo realizado en el año 2002, contaba con 6 personas por cada $\mathrm{km} 2$. Ocupaba un área de $12.147 \mathrm{~km} 2$ y está dividido en 16 distritos. La capital del Departamento es Pilar.

Entre los años 1962 y 1972 la población aumentó en más de 15.000 personas; en el periodo 1972-1982 el volumen poblacional se vio afectado por el desprendimiento del distrito Yabebyry, que actualmente pertenece al departamento Misiones. Luego de experimentar una leve disminución entre 1982 y 1992, la población se incrementó en un 
poco más de 6.500 habitantes en la última década. Actualmente representa el 1,5\% del total poblacional del país.

Según el censo 2002, residían en Ñeembucú 76.348 personas y es uno de los pocos departamentos que albergaba a la mayoría de sus habitantes en área urbana, aunque la diferencia con la rural era mínima. Existía una leve mayoría de varones. A nivel país presentaba la mayor proporción de adultos mayores, aunque respecto de los demás grupos etáreos el porcentaje de esta parte de la sociedad era el menor; los adultos y niños conformaban las agrupaciones de más volumen, y los jóvenes constituían una fracción un tanto inferior. La cantidad de personas con partida de nacimiento y cédula de identidad, en relación al total departamental, es la mayor entre las divisiones político administrativas de la nación.

La topografía del departamento se caracteriza por el amplio predominio de zonas planas y bajas. Este aspecto del territorio favorece la existencia de grandes esteros y pantanos que son intransitables en época de lluvias, además de producir la inundación de extensas superficies a causa de las crecientes de los ríos Paraguay, Tebicuary y Negro.

Las partes altas, en proporción mucho menor que las bajas, son discontinuas y forman áreas en las que se asientan las poblaciones, se cultiva la tierra y se cría el ganado vacuno. El departamento cuenta con numerosos lugares históricos. Pueden mencionarse, por ejemplo, Paso de Patria e Itá Pirú, donde se libraron duelos de artillería entre las baterías paraguayas y la escuadra del Brasil. El Banco de Itá Pirú, Estero Bellaco, Humaitá, Curupayty, Sauce y Boquerón son sitios en los que se desarrollaron memorables batallas durante la guerra de la Triple Alianza. También se encuentra en la zona, San Fernando, lugar donde el Mariscal Francisco Solano López asentó su campamento y en el que hasta hoy se observan vestigios de las trincheras paraguayas.

En cuanto a educación, se menciona en el material que a pesar del aumento poblacional en el periodo 1992-2002, la cantidad de estudiantes matriculados en primaria prácticamente se mantuvo en este lapso, sin embargo, en la secundaria, duplicó. Al tiempo de la realización del censo (2002), asistían a alguna institución de enseñanza formal poco más del 30\% de la población de 7 años y más de edad, y el alfabetismo abarcaba a casi el $90 \%$ de las personas de 15 años y más. 
En esa última década la cantidad de locales de primaria y secundaria aumentó su ritmo de crecimiento, mientras que el número de cargos docentes en el nivel primario mantuvo su velocidad de progresión.

En cuanto a la salud, entre los años 1972 y 2002 según el informe, se triplicó la cantidad de centros de salud y puestos sanitarios, y en ese mismo periodo el número de camas por cada 10.000 habitantes casi se cuadruplicó, siendo al tiempo del censo, el mayor a nivel departamental.

En cuanto a la economía, aunque de 1972 a 1992 no hubo mucha variación en la cantidad de personas económicamente activas, se observa un aumento importante entre 1992 y 2002, pese a que en este periodo la tasa de ocupados ha tenido una leve disminución.

Respecto a la vivienda, según el censo (2002), residen en promedio 4 personas por vivienda, de un total de 19.355 viviendas particulares ocupadas. En comparación a décadas anteriores, la cobertura de servicios básicos ha aumentado significativamente.

\section{Universidad Nacional de Pilar}

\section{Antecedentes}

Según consta en el Estatuto de la Universidad (2015), la Universidad Nacional de Pilar se encuentra ubicada en la eco región del Ñeembucú, vértice sur de la República del Paraguay, tierra heroica y legendaria. Reunidos en Asamblea Universitaria por voluntad de todos sus estamentos; convencidos de que la educación es un compromiso social y uno de los elementos fundamentales de la democracia, el desarrollo sostenible y la paz; interpretando las raíces históricas de la UNP y su identidad definida por la voluntad popular expresada en su génesis en el año 1991.

La Universidad Nacional de Pilar, es una institución de Derecho Público, autónoma, autárquica, con presencia jurídica y patrimonio propio, que se rige por la Constitución Nacional, las leyes y su Estatuto. Concibe a la educación superior como un bien público, un derecho fundamental de los habitantes de la República del Paraguay, no se adscribe a ninguna corriente ideológica, es laica y garantiza la libertad de investigación y expresión y desarrolla su misión sin discriminación de orden étnico, cultural, lingüístico, religioso, de nacionalidad, ideológica, opinión política o gremial, sexo, posición económica, condición social o caracteres físicos. No se desvincula de los problemas ideológicos, políticos o sociales del país y del mundo, sino que los estudia científicamente. Fija su 
domicilio legal y sede central en la ciudad de Pilar, capital del Departamento de Neembucú.

\section{Objetivos}

- Organizar el gobierno universitario consolidando su autonomía plena;

- Garantizar la participación ciudadana, el pluralismo de ideas y el ejercicio de los derechos en un orden solidario, justo, libre e igualitario;

- Defender los derechos humanos; garantizar la calidad en la formación profesional, la investigación científica y la extensión universitaria;

- Propender al crecimiento armónico de la universidad, custodiando su área de influencia, preservando el ambiente y el patrimonio histórico y cultural del departamento y de la Nación;

- Impulsar la integración con las universidades de la región, el país y el mundo, reconociendo a la educación superior como un bien público social, un derecho humano universal y un deber del Estado, invocando la protección de Dios y nuestras convicciones democráticas. (Estatuto UNP. 2015)

\section{Fines de la Universidad}

- El desarrollo de la personalidad humana, inspirado en los valores de la democracia, la libertad, la justicia social y el respeto a los derechos humanos

- La enseñanza y la formación profesional superior orientadas al desarrollo humano, que contribuyan al empoderamiento social;

- La investigación científica en las diferentes áreas del saber humano preferentemente en aquellas que favorezcan al desarrollo nacional desde una perspectiva crítica;

- El fomento y la difusión de la cultura universal y, en particular, de la local y nacional;

- La extensión de los conocimientos, los servicios, la cultura a la sociedad través de actividades del aprendizaje recíproco.

- El estudio sistémico de la realidad nacional, regional y universal proponiendo alternativas de solución a los problemas humanos y socio ambientales. (Estatuto UNP. 2015).

\section{Visión}

- La Universidad se propone como visión ser una "Institución de Educación Superior, referente por su excelencia académica, reconocida por la producción científica y tecnológica en el ámbito local, nacional e internacional, con una gestión participativa 
e innovadora, a través de talentos humanos comprometidos y éticos, en interacción con el contexto social y ecológico”. (Plan líneas estratégicas UNP. 2015)

\section{Misión.}

- Se propone como misión "Formar profesionales críticos, creativos, con principios éticos, conocimientos científicos, capacidad tecnológica y artística como ciudadanos protagonistas en una institución inclusiva, solidaria e internacionalizada, comprometida con el desarrollo sostenible mediante la docencia, la investigación, la tecnología y la extensión”. (Plan líneas estratégicas UNP. 2015)

\section{Líneas estratégicas.}

La Universidad define 4 líneas estratégicas que se hallan establecidas en el Plan Estratégico Institucional UNP 2016 - 2020.

a) Excelencia en la Educación Superior, mediante el cumplimiento de sus funciones esenciales: Docencia, investigación y extensión. La dimensión académica está basada en la autonomía institucional, en la libertad de cátedra, enfocada en los criterios interdisciplinarios y la orientación pedagógica que promueva el pensamiento crítico, la creatividad y los principios éticos, de tal modo a contribuir en la formación de profesionales con ejercicio pleno de la ciudadanía y comprometidos con los valores universalmente aceptados, dispuestos a la defensa de los derechos humanos, de los derechos laborales, de un ambiente sostenible. La investigación reconocida como el proceso dinámico de producción de conocimiento y desarrollo tecnológico en diferentes áreas; orientada a brindar alternativas de solución a situaciones problemáticas de índole social y ambiental de tal modo a realizar contribuciones relevantes al desarrollo socioeconómico del departamento y ámbito de influencia de la UNP y la extensión que constituye la interacción con la comunidad; a través de programas y proyectos implementados que tiendan a la construcción de redes de aprendizaje entre docentes, estudiantes, ciudadanía y que contribuyan al buen vivir.

b) Interacción con el contexto social y ecológico: esta línea estratégica es distintiva en la misión institucional, la priorización de la situación socio-ecológica debe ser transversal en las funciones esenciales de la Educación Superior: la docencia, investigación y extensión. Mediante la misma se pretende promover soluciones alternativas a la realidad departamental y regional, de manera que la institución sea referente en el tema ecológico, histórico, social y artístico. 
c) Relaciones interinstitucionales e internacionales: Cooperación e internacionalización. Considerando las tendencias actuales en cuanto a internacionalización, la UNP define su ámbito de actuación vinculado a los demás actores sociales y culturales (públicos y privados, nacionales e internacionales, organizaciones sociales y empresariales) a efectos de fortalecer los lazos de cooperación que se transformen en acciones concretas y permitan desplegar y aprovechar todas las posibilidades de contribución mutua en el desarrollo de la institución y de la sociedad.

d) Gestión y Gobierno: debe basarse en el cogobierno para generar una gestión institucional inclusiva, participativa, solidaria e innovadora, concordante con una estructura administrativa flexible y dinámica, que responda a las demandas internas y externas, basado en el principio de centralización normativa y descentralización operativa, que desarrolle un sistema integrado de comunicación e información de la gestión académica, de investigación y extensión, que facilite una información actualizada y confiable para la toma de decisiones en los niveles estratégicos y operativos.

\section{Agenda 2030 para el Desarrollo Sostenible}

\section{Antecedentes.}

Los antecedentes de la Agenda 2030 para el desarrollo sostenible publicado por la Cancillería de Colombia a través de la página web (https://www.cancilleria.gov.co), se detallan a continuación.

\section{Río +20: punto de partida para la nueva agenda del desarrollo global.}

Durante la Conferencia de las Naciones Unidas sobre desarrollo sostenible Río +20, se adoptó el documento de resultados "El futuro que queremos", el cual reconoció "la importancia y utilidad de desarrollar un conjunto de objetivos de desarrollo sostenible ODS-, basados en Agenda 21, el Plan de Implementación de Johannesburgo y los Principios de Río que incorporen de forma equilibrada las tres dimensiones del desarrollo sostenible y sus interrelaciones, y sean coherentes con la agenda de las Naciones Unidas para el desarrollo posterior a 2015”.

Así se adoptó el concepto de los ODS, incluyendo las principales características presentadas por Colombia: orientados a la acción, ser concisos y fáciles de comunicar, limitados en número, tener un carácter global y ser universalmente aplicables a todos los países, teniendo en cuenta las diferentes realidades y capacidades nacionales. 
Se estableció un proceso intergubernamental inclusivo y transparente para formular los ODS en el marco de la Asamblea General. Se acordó crear un Grupo Abierto de Trabajo compuesto por 30 representantes de los cinco grupos regionales de las Naciones Unidas para lograr una representación geográfica equitativa y equilibrada. Colombia fue miembro del grupo de trabajo. En la práctica 70 países participaron en el grupo y comparten los 30 puestos. Colombia lo hizo con Guatemala.

El grupo de trabajo presentó un informe a la Asamblea General de Naciones Unidas en septiembre 2014, con una propuesta de objetivos de desarrollo sostenible para que esta los examinara y adoptara las medidas adecuadas.

\section{El marco institucional para el desarrollo sostenible (IFSD).}

Para seguir avanzado hacia la consolidación de los ODS, la Comisión de las Naciones Unidas sobre el Desarrollo Sostenible (CDS- Comission on Sustainable Development) fue reemplazada por un Foro Político de Alto Nivel, que proveerá lineamientos y recomendaciones en materia de desarrollo sostenible. Este se reunió por primera vez el 24 de septiembre de 2015 en el marco del 68 periodo de Sesiones de la Asamblea General de la ONU.

Se acordó fortalecer el Consejo Económico y Social - ECOSOC- y se reafirmó la importancia del mismo como órgano principal para la revisión de políticas diálogo y formulación de recomendaciones en materia de desarrollo social y económico, y el seguimiento de los Objetivos de Desarrollo del Milenio.

\section{Objetivos de Desarrollo del Milenio a los Objetivos de Desarrollo Sostenible.}

La agenda del desarrollo post 2015 hace referencia a aquellas iniciativas/mecanismos/procesos que se han puesto en marcha para definir la agenda internacional del desarrollo sostenible posterior a 2015, periodo de cumplimiento de los Objetivos de Desarrollo del Milenio (ODM).

La Asamblea General de Naciones Unidas recientemente adoptó la Resolución 68/309 mediante la cual decide que la propuesta de Objetivos contenida en el Informe del Grupo Abierto de Trabajo será la base principal para integrar dichos objetivos en la Agenda de Desarrollo Post-2015.

\section{Objetivos de Desarrollo Sostenible.}

Los Objetivos de desarrollo sostenible son el plan maestro para conseguir un futuro sostenible para todos. (www.un.org). 
Son 17 los objetivos de Desarrollo sostenible, con los cuales se pretende transformar el mundo.

Fin de la pobreza; Hambre Cero; Salud y bienestar; Educación de calidad; Igualdad de género; Agua limpia y saneamiento; Energía asequible y no contaminante; Trabajo decente y crecimiento económico; Industria, innovación e infraestructura; Reducción de las desigualdades; Ciudades y comunidades sostenibles; Producción y consumo responsables; Acción por el clima; Vida submarina; Vida de ecosistemas terrestres; Paz, justicia e instituciones sólidas; Alianzas para lograr los objetivos. (www.un.org).

\section{Objetivo 4 Educación de Calidad.}

- El objetivo 4 habla de garantizar una educación inclusiva, equitativa y de calidad y promover oportunidades de aprendizaje durante toda la vida para todos. (www.un.org)

\section{Metas del objetivo 4.}

- El objetivo 4 de Desarrollo Sostenible contempla una serie de metas, de los cuales la meta 4.3 hace referencia a la educación superior

- 4.1 De aquí a 2030, asegurar que todas las niñas y todos los niños terminen la enseñanza primaria y secundaria, que ha de ser gratuita, equitativa y de calidad y producir resultados de aprendizajes pertinentes y efectivos.

- 4.2 De aquí a 2030, asegurar que todas las niñas y todos los niños tengan acceso a servicios de atención y desarrollo en la primera infancia y educación preescolar de calidad, a fin de que estén preparados para la enseñanza primaria.

- 4.3 De aquí a 2030, asegurar el acceso igualitario de todos los hombres y las mujeres a una formación técnica, profesional y superior de calidad, incluida la enseñanza universitaria

- 4.4 De aquí a 2030, aumentar considerablemente el número de jóvenes y adultos que tienen las competencias necesarias, en particular técnicas y profesionales, para acceder al empleo, el trabajo decente y el emprendimiento

- 4.5 De aquí a 2030, eliminar las disparidades de género en la educación y asegurar el acceso igualitario a todos los niveles de la enseñanza y la formación profesional para las personas vulnerables, incluidas las personas con discapacidad, los pueblos indígenas y los niños en situaciones de vulnerabilidad. 
- 4.6 De aquí a 2030, asegurar que todos los jóvenes y una proporción considerable de los adultos, tanto hombres como mujeres, estén alfabetizados y tengan nociones elementales de aritmética

- 4.7 De aquí a 2030, asegurar que todos los alumnos adquieran los conocimientos teóricos y prácticos necesarios para promover el desarrollo sostenible, entre otras cosas mediante la educación para el desarrollo sostenible y los estilos de vida sostenibles, los derechos humanos, la igualdad de género, la promoción de una cultura de paz y no violencia, la ciudadanía mundial y la valoración de la diversidad cultural y la contribución de la cultura al desarrollo sostenible

- El Instituto de Estadística de la UNESCO supervisa oficialmente la meta 4.3, mediante el indicador de "la tasa bruta de matrícula en la educación superior". (www.un.org)

\section{El papel estratégico de la Educación Superior en el Desarrollo Sostenible de América}

\section{Latina y el Caribe.}

- El Plan de acción de la CRES (2018), establece lineamientos, y específicamente el "lineamiento 1", habla del papel estratégico de la educación superior en el desarrollo sostenible y menciona que el desarrollo sostenible es una forma de pensamiento que proyecta al hombre en una mejor relación con el contexto, un imperativo categórico que establece el valor ético de la convivencia en el mundo. Su desempeño debe responder a las demandas sociales que requieran conocimientos técnicos, eficientes, oportunos y cuya aplicabilidad apunten hacia la superación de los conflictos y situaciones de afectación social para alcanzar el bienestar común. Esta fundamentación establece el pensamiento sostenible como una construcción epistémica de las ciencias sociales, integrada, compleja, interdisciplinaria, universal y transformadora de alto contenido económico, social y ambiental para enfrentar la crisis civilizatoria. Se fundamenta en la interpretación de la realidad de los hombres con enfoques multidimensionales de sus problemas para intervenir de acuerdo con la gestión de riesgo en términos de una mejor convivencia. Por ello sus principios se basan en el humanismo, en el bien público, derecho humano y la base para garantizar la realización de otros derechos.

- La educación está inmersa en valoraciones éticas, por ello sociedad y educación se articulan en una construcción indisoluble del pensamiento social y complejo que se expresa a través de los objetivos de desarrollo sostenible, y particularmente con el objetivo 4, de la agenda de educación. (CRES. 2018) 


\section{ESTRATEGIAS METODOLÓGICAS}

\section{Objetivo Principal de la investigación}

* Determinar de qué manera la Universidad Nacional de Pilar, como Institución de Educación Superior, contribuye al desarrollo sostenible en el Departamento de Ñeembucú, desde sus funciones sustantivas, atendiendo su visión, misión y política institucional.

\section{Objetivos Específicos.}

Conocer el sistema de acceso a la Universidad Nacional de Pilar, en concordancia con su naturaleza y la Agenda 2030.

* Determinar la contribución de la Universidad en relación a la enseñanza y la formación profesional superior orientada al desarrollo de las personas y su contexto social.

* Conocer los proyectos de investigación científica emprendidos en la universidad en el contexto de desarrollo sostenible.

* Determinar de qué manera la universidad como institución de educación superior se vincula con la sociedad desde su política institucional y pertinencia social para el desarrollo sostenible en el departamento de Ñeembucú.

\section{Tipo y método de estudio}

La investigación responde a un estudio de caso, implementa un tipo de estudio descriptivo, explicativo y de corte transversal.

La investigación implementa el enfoque mixto: cualitativo y cuantitativo.

La investigación cualitativa proporciona profundidad a los datos, dispersión, riqueza interpretativa, contextualización del ambiente o entorno, detalles y experiencias únicas. La investigación cuantitativa ofrece la posibilidad de generalizar los resultados más ampliamente, otorga control sobre los fenómenos, así como un punto de vista basado en conteos y magnitudes. También, brinda una gran posibilidad de repetición y se centra en puntos específicos de tales fenómenos, además de que facilita la comparación entre estudios similares. (Hernández Sampieri, 2014, pág. 16,17).

\section{Población y muestra}

La población está conformada por Directivos de la Universidad Nacional de Pilar, representantes gubernamentales, departamentales y de empresas. 
La muestra estará conformada por el Rector de la Universidad, Directores Generales y de las diferentes facultades, Gobernador, Intendente, Empresarios.

\section{Técnicas de recolección de datos.}

Se implementará como técnicas de recolección de datos.

- Las entrevistas semi estructurada, aplicadas a Directivos de la Universidad (directores de investigación, extensión, académica y administrativa), representantes gubernamentales, departamentales, y de empresas)

- Revisión documental: revisión de proyectos, actividades realizadas y documentadas.

\section{RESULTADOS Y CONCLUSIONES PROVISIONALES}

Los resultados provisionales, resultante de las informaciones recabas mediante los instrumentos de recolección de datos como los cuestionarios, las entrevistas y el análisis documental, indican cual importante es la estadía de la Universidad Nacional de Pilar en el Departamento de Neembucú y el cumplimiento con su compromiso social y desarrollo sostenible a través de sus funciones sustantivas como Institución de Educación Superior. La Universidad Nacional de Pilar tiene un papel estratégico en el desarrollo sostenible del Departamento de Neembucú, generando conocimientos, científicos, tecnológicos y social, formando profesionales y contribuyendo en la búsqueda y propuestas de mejoramiento de la calidad de vida de sus pobladores.

Se puede mencionar que la UNP, cumple con su Misión, de formar profesionales críticos, creativos, con principios éticos, conocimientos científicos, capacidad tecnológica y artística como ciudadanos protagonistas en una institución inclusiva, solidaria e internacionalizada, comprometida con el desarrollo sostenible mediante la docencia, la investigación, la tecnología y la extensión.

El sistema de acceso establecido garantiza una educación inclusiva, equitativa y de calidad y promover oportunidades de aprendizaje durante toda la vida para todos.

Los principales requisitos para el Ingreso a la Universidad Nacional de Pilar son: Título de Bachiller o equivalente registrado y legalizado en la UNP, Certificados de Estudios registrado y legalizado en la UNP, Cumplir los demás requisitos establecidos en los Reglamentos de la Universidad Nacional de Pilar y de sus respectivas Facultades.

La Universidad Nacional de Pilar contribuye al desarrollo sostenible a través de la Docencia, la Investigación y la Extensión o vinculación con la sociedad 
La Universidad cumple con los objetivos de Desarrollo Sostenible, en especial el Objetivo 4 "Educación de Calidad" y sus metas.

Por último mencionar el papel fundamental y más importante de la Universidad Nacional de Pilar a través de su Rector y Senador Dr. Víctor Ríos, quien ha presentado la propuesta y promovido la aprobación de la gratuidad de la educación universitaria, la cual ha sido aprobado por Ley $\mathrm{N}^{\mathrm{o}} 6628$ que establece "la gratuidad de los cursos de admisión y de grado en todas las Universidades Públicas del país, en el Instituto Superior de Bellas Artes, Instituto Nacional de Educación Superior, Institutos de Formación Docente, dependientes del Ministerio de Educación Media de Instituciones educativas del sector oficial o instituciones educativas del sector subvencionado. La gratuidad, se extiende de igual manera, a quienes se encuentren en situación de vulnerabilidad".

\section{LISTA DE REFERENCIAS}

Atlas Censal del Paraguay. Dirección General de Estadística y Censo. (2002). Neembucú. Accedido

file:///C:/Users/asus/Downloads/Documents/15\%20Atlas\%20Neembucu\%20cen so.pdf

Banco Mundial. (2018).Informe sobre el desarrollo mundial 2018. Aprender a hacer realidad la promesa de la educación, cuadernillo del Panorama General. Accedido en www.bancomundial.org

Comisión Mundial sobre el Medio Ambiente y el Desarrollo. (1987). Definición de desarrollo sostenible. Accedido en file://C:/Users/asus/Downloads/Documents/CMMAD-Informe-ComisionBrundtland-sobre-Medio-Ambiente-Desarrollo.pdf

CRES. (2018). Plan de acción. 2018- 2028. III Conferencia Regional de Educación Superior para América Latina y el Caribe. El Papel estratégico de la Educación Superior en el desarrollo sostenible de América Latina y el Caribe.

Hernández Sampieri, R. (2014). En M. d. edición.. Méjico: McGRAW-HILL / INTERAMERICANA EDITORES, S.A. DE C.V.

Ibáñez, B. C. (1994). Pedagogía y Psicología Interconductual. Revista Mexicana de Análisis de la Conducta,

Naciones Unidas. (1987, pág. 23). Informe Brundtland. Comisión Mundial sobre el Medio Ambiente y el Desarrollo. 
Pontificia Universidad Javeriana (2018). Normas APA. Sexta Edición.

Programa de las Naciones Unidas para el Desarrollo (2015) Agenda 2030. Accedido en https://www.undp.org/content/undp/es/home/sustainable-developmentgoals/goal-4-quality-education.html

Ramírez S., Rutilia. (2012). La Educación Superior para el Desarrollo Sostenible. Coloquio Internacional de Gestión Universitaria. Accedido en https://repositorio.ufsc.br

UNESCO. (2015). Agenda para el Desarrollo Sostenible. Antecedentes. Accedido en https://www.cancilleria.gov.co)

Universidad Nacional de Pilar (2015). Estatuto.

Universidad Nacional de Pilar (2015). Plan Operativo Institucional.

Universidad Nacional de Pilar. Resolución Nº 14. (2019). Reglamento para optar al título de postgrado en la Universidad Nacional de Pilar. 